\title{
Physiological fluctuations in white matter are increased in Alzheimer's disease and correlate with neuroimaging and cognitive biomarkers
}

Ilia Makedonov ${ }^{1,5}$ MHSc, J Jean Chen $2,3,5$ PhD, Mario Masellis ${ }^{4,6}$ MD, PhD, Bradley J Maclntosh ${ }^{3,5,6} \mathrm{PhD}$, for the Alzheimer's Disease Neuroimaging Initiative

${ }^{1}$ Faculty of Medicine, University of Toronto, Toronto, Ontario, Canada

${ }^{2}$ Rotman Research Institute, Baycrest Centre for Geriatric Care, University of Toronto, Toronto, Ontario, Canada

${ }^{3}$ Medical Biophysics, University of Toronto, Toronto, Ontario, Canada

${ }^{4}$ Division of Neurology, Department of Medicine, Sunnybrook Health Sciences Centre, University of Toronto, Toronto, Ontario, Canada

${ }^{5}$ Heart and Stroke Foundation Canadian Partnership for Stroke Recovery, Sunnybrook Research Institute, Toronto, Ontario, Canada

${ }^{6}$ Brain Sciences, Sunnybrook Research Institute, Toronto, Ontario, Canada

\section{Corresponding author}

Bradley J MacIntosh

Sunnybrook Research Institute

2075 Bayview Avenue, Room M6-180

Toronto, Ontario, Canada, M4N 3M5

Email: bmac@sri.utoronto.ca

Phone: $4164806100 \times 7277$ 


\section{Abstract}

The objective of this study was to determine whether physiological fluctuations in white matter (PFwm) on resting state functional magnetic resonance images ( $r s-f M R I)$ could be used as an index of neurodegeneration and Alzheimer's disease (AD). Using rs-fMRI data from participants in the Alzheimer's Disease Neuroimaging Initiative (ADNI), PFwm was compared across cohorts: cognitively healthy, mild cognitive impairment (MCI), or probable AD. Secondary regression analyses were conducted between PFwm and neuroimaging, cognitive and cerebrospinal fluid (CSF) biomarkers. There was an effect of cohort on PFwm ( $t=5.08$, $d f=424, p<5.7 e-7)$, after accounting for nuisance effects from head displacement and global signal ( $t>6.16)$. From the neuroimaging data, PFwm was associated with glucose metabolism $(t=-2.93, d f=96, p=0.004)$, but not ventricular volume $(p<0.49)$ or hippocampal volume $(p>0.44)$. From the cognitive data, PFwm was associated with composite memory $(t=-3.24$ [df=149], $p=0.0015)$ but not executive function $(p>0.21)$. PFwm was not associated with CSF biomarkers. In one final omnibus model to explain PFwm ( $\mathrm{N}=124)$, glucose metabolism $(p=0.04)$ and cohort $(p=0.008)$ remained significant, as were global and head motion RMS terms, whereas memory was not $(p=0.64)$. PFwm likely reflects end-arteriole intracranial pulsatility effects that may provide additional diagnostic potential in the context of $A D$ neurodegeneration.

Keywords: Alzheimer's disease; neurodegeneration; physiological noise; pulsatility; resting state functional MRI; white matter. 


\section{Introduction}

Alzheimer's disease $(A D)$ is the most common neurodegenerative disorder and is characterized by progressive cognitive dysfunction that interferes with daily activities and results in significant morbidity. Mild cognitive impairment $(\mathrm{MCl})$ is considered the prodromal stage of Alzheimer's dementia that can predate AD by several years ${ }^{1,2}$. Although the $\mathrm{MCl}$ period provides a potential intervention window, there are no established treatments.

Furthermore the $\mathrm{MCl}$ classification is increasingly viewed as heterogeneous since not all $\mathrm{MCl}$ individuals convert to $A D^{3,4}$.

AD-related progression is influenced by multiple pathophysiological changes, notably amyloid deposition ${ }^{5}, 6$ cerebral amyloid angiopathy ${ }^{7}$, and new evidence of $A D$ pathogenesis within pericytes ${ }^{8}$. Increasingly, cerebrovascular techniques are used to characterize small vessel disease phenomena that contribute to neurodegeneration ${ }^{9}$. Central and cerebral hemodynamic measures, such as arterial stiffening, carotid artery stiffening and cerebral blood flow are predictors of cognitive performance ${ }^{10}$, cognitive decline ${ }^{11}$ and $A D$ diagnosis ${ }^{12,13}$. Work by others provides support for the theory of mechanical factors of arterial aging ${ }^{14}$, which leads to excessive intracranial pulsatility (described as windkessel dysfunction) in normal and demented older adult cohorts ${ }^{15,16}$.

In the current study we use a summary measure of physiological noise in white matter, which we reported on previously in a group with small vessel disease ${ }^{17}$, to serve as a proxy index of cerebrovascular dysfunction, whereby elevated arterial pulsatility contributes to increased image temporal variance observed in white matter on resting state functional MRI (rs-fMRI). Rs-fMRI has traditionally been used in AD to study grey matter functional connectivity, such as reported decreased co-activation of default mode network ${ }^{18}$. Whereas white matter rsfMRI signals are viewed as nuisance variables in grey matter connectivity analyses ${ }^{19,20}$, they 
also provide non-neuronal physiological contrast, due to: cardiac pulsatility ${ }^{17,21,22}$, vasomotion in ischemia ${ }^{23}$, and cerebrovascular impairment ${ }^{24}$. To date there are but a few rs-fMRI studies that specifically investigate white matter ${ }^{17,25}$, therefore our primary objective is to compare PFwm among normal older controls, $\mathrm{MCl}$ and $\mathrm{AD}$ groups. We hypothesize that there will be an effect of group on PFwm from among other factors that can influence this rs-fMRI summary measure. Our secondary objective was to determine whether PFwm was associated with any established markers of AD pathology and/or neurodegeneration. For this secondary objective, neuroimaging, cognitive and cerebrospinal fluid (CSF) data are considered. For neuroimaging markers, we used glucose metabolism, ventricular volume and hippocampal volume. The former reflects altered bioenergetics reported in $A D^{26}$, whereas the latter two are structural markers of neurodegeneration and $A D{ }^{27,}, 28$. For the cognitive measures, composite scores of memory and executive function, developed from ADNI, were considered ${ }^{29}$. Lastly, the following AD pathological markers were also considered from CSF: amyloid-beta 1 to 42 peptide, tau and phosphorylated tau protein levels ${ }^{30}$. Secondary analyses were conducted in this manner (i.e. separately) to capture the largest sub-sample size for each of the association tests.

\section{Methods}

\subsection{ADNI dataset}

Data were obtained from the ADNI database (adni.loni.usc.edu). ADNI was launched in 2003 by the National Institute on Aging, the National Institute of Biomedical Imaging and Bioengineering, the Food and Drug Administration, private pharmaceutical companies and non-profit organizations. The primary goal of ADNI has been to test whether serial MRI, PET, other biological markers, and clinical/neuropsychological assessment can be combined to 
measure the progression of $\mathrm{MCl}$ and early $\mathrm{AD}$. ADNI is the result of efforts of many coinvestigators from a broad range of academic institutions and private corporations, and participants have been recruited from over 50 sites across the U.S. and Canada.

\subsection{Ethics statement}

Participants gave written informed consent at the time of enrollment and completed questionnaires approved by each participating site's Institutional Review Board (IRB).

\subsection{Participants}

Only data from ADNI-2 and ADNI-GO were used since ADNI-1 did not include rs-fMRI.

Participants with rs-fMRI within the first year of participation in the study were selected, which amounted to $437 \mathrm{rs}-\mathrm{fMRI}$ datasets for the primary objective. First-year data were selected to to minimize potential retention bias from repeat scans. Available rs-fMRI and corresponding structural T1 images were acquired between June 302010 and May 122014 (downloaded in June 2014). Sample sizes for the secondary objectives were smaller due to the inclusion of rs-fMRI with other measures (i.e. matched samples with neuroimaging, cognitive, or CSF data).

\subsubsection{Normal Controls, $\mathrm{MCl}$ and $\mathrm{AD}$ criteria}

Participants were enrolled if they were between 55-90 years old, spoke English or Spanish as the first language, completed at least 6 years of schooling. Diagnostic classification was made by $A D N I$ investigators using established criteria ${ }^{31-33}$, albeit early and late $\mathrm{MCl}$ group were combined into a single $\mathrm{MCl}$ group. Controls had mini-mental status examination (MMSE) $)^{34}$ scores between 24-30 and no significant memory concerns. MCI adults had: MMSE scores between 24-30; memory complaint; objective memory loss as quantified by the Wechsler Memory Scale Logical Memory II test ${ }^{35}$; a Clinical Dementia Rating (CDR) ${ }^{36}$ score of 0.5 ; lack of cognitive impairment in other domains such as executive function, visuospatial function and 
language; relative sparing of activities of daily living; and absence of frank dementia.

Participants in the AD cohort fulfilled the NINCDS-ADRA criteria for probable AD.

\subsection{MRI acquisition}

MR images were collected on 3 Tesla Philips MRI systems (Philips, Amsterdam, Netherlands) from a total of 13 sites using a standardized protocol ${ }^{37}$. Data from 3 Tesla MRI systems were considered (and not 1.5 T) because physiological sources in fMRI are field-strength dependent ${ }^{38}$. T1 images were acquired with an echo time (TE) of $3 \mathrm{~ms}$, a repetition time (TR) of $7 \mathrm{~ms}$, flip angle of $9^{\circ}$, slice thickness of $1.2 \mathrm{~mm}$ and a matrix size of $256 \times 256 \times 170$. Rs-fMRI echo planar images were acquired with a TE of $30 \mathrm{~ms}$, TR of $3000 \mathrm{~ms}$, flip angle of $80^{\circ}$, slice thickness of $3.3 \mathrm{~mm}$ and a matrix size of $64 \times 64 \times 48$ (note: $<5 \%$ of rs-fMRI data were collected with a TR of $2250 \mathrm{~ms}$ ).

\section{$2.5 \mathrm{MRI}$ preprocessing}

Preprocessing of the structural T1 images involved bias field correction using a histogram peak-sharpening algorithm (N3) ${ }^{39}$. Echo planar rs-fMRI were corrected for head motion using mcflirt, a tool that is part of the FMRIB Software Library (FSL) ${ }^{40}$. Next, the mean relative root mean squared (RMS) head displacement $(\mathrm{mm})$ was determined from FSL's mcflirt and used as a nuisance regressor. In addition, a global rs-fMRI signal from within-skull voxels (i.e. brain parenchyma and ventricles) was also used as a second nuisance regressor, extracted from the global signal time series. Global RMS in units of signal intensity was then calculated on the demeaned global time series. The rs-fMRI preprocessing steps were chosen based on the following rationale: 1) white matter signals tend to be spatially consistent and robust in rsfMRI ${ }^{41}$, hence the appeal of a PFwm summary measure, 2) the use of voxel-wise regression of motion traces may not be indicated for this application as it may introduce spatially nonuniform effects across the white matter ${ }^{42}$ and 3 ) both global RMS and head displacement 
RMS can be viewed as confound effects that could each contribute to PFwm, of which case the latter is reported as strongly related to frame-to-frame change in signal ${ }^{42,43}$. Head displacement and global RMS regressors were included in the group-level model for all analyses (see Statistics below).

Previously we have shown that PFwm correlates with cardiac pulsatility in white matter. We confirmed our findings by comparing conventional rs-fMRI and high temporal resolution rsfMRI (TR of $250 \mathrm{~ms}$ ) in young and older adults, demonstrating that aliasing of the cardiac signal in conventional fMRI data did not significantly influence this outcome ${ }^{17}$. In the current study, PFwm was calculated in native rs-fMRI space using a similar method to the previous report ${ }^{17}$. Step 1 : isolate per voxel (i.e. $\left.x, y, z\right)$ physiological fluctuations in the rs-fMRI time series by computing the following equation:

$$
P F_{x, y, z}=\sqrt{\sigma_{x, y, z}^{2}-\sigma_{\text {thermal }}^{2}}
$$

Thermal noise variance $\left(\sigma^{2}\right.$ thermal) was determined as the average variance in a user-defined region of interest $(\mathrm{ROI})$ placed outside the brain in a corner of each resting-state image. The placement of the background $\mathrm{ROI}$ was chosen to minimize contribution from EPI ghosting artefact and evaluated by histogram inspection. Step 2: the physiological noise from step 1 was normalized by the mean signal intensity in each voxel, producing a fractional blood oxygenation level dependent (BOLD) change map (see examples in Figure 1). Step 3: PFwm was calculated as the mean physiological noise across WM voxels and used as a summary metric (see below on PFwm adjustment for head motion). To facilitate quality control by visual inspection, PFwm white matter maps were registered to a common template space, by affine transformation and by using the $\mathrm{T} 1$ structural image as an intermediate transformation. The common template was created by randomly selecting 15 participants from each cohort (45 in 
total) and co-registering the T1 images to a common template using Advanced Normalization Tools. T1 images were brain-extracted and segmented into CSF, grey matter and WM tissue classes. White matter masks were eroded using a 3mm Gaussian kernel. The medulla, pons, midbrain and cerebellum were removed from rs-fMRI to focus the analysis on white matter found in the telencephalon.

\subsection{Secondary analyses on biomarker measures}

Ventricular volume, hippocampal volume and FDG glucose metabolism were used as neuroimaging biomarkers. Composite scores of memory and executive function and protein levels in CSF were ascertained from the ADNI database. ADNI ventricular volumes were calculated as described by Nestor and colleagues ${ }^{27}$. Both ventricular volume and hippocampal volume were normalized by total intracranial volume. For the FDG glucose metabolism intensity normalized images, a composite grey matter region of interest mask was used to generate a single glucose metabolism value from five regions identified by Landau et al. ${ }^{26}$ (i.e. left and right angular gyri, left and right inferior temporal gyri and bilateral posterior cingulate cortex). Cognitive composite scores for executive function and memory were computed by Crane et al. ${ }^{29}$ using factor analysis. CSF protein concentrations $(\mathrm{pg} / \mathrm{mL})$ were calculated by the University of Pennsylvania ADNI Biomarker Laboratory, as described in the work of Shaw and colleagues ${ }^{30}$.

\subsection{Statistics}

Primary and secondary analyses were conducted using R (version 3.1.2 from RStudio Inc). The primary analysis used a univariate linear model (ANOVA, type III) and included the following independent variables: cohort, age, visit, WM volume, head displacement RMS, global RMS and site, of which case cohort was the primary variable of interest. For secondary analyses involving sub-samples, PFwm (adjusted for global and head RMS terms) was 
compared against ventricular volume, hippocampal volume and glucose metabolism. Adjusted PFwm was also compared with composite measures of memory and executive function. Adjusted PFwm was compared with protein levels in CSF. The final regression test was performed using all variables that were found to correlate with PFwm, with specific interest in the effect of cohort on PFwm.

\section{Results}

Characteristics of the sample drawn from ADNI are summarized in Table 1. There were cohort differences that included: MMSE scores $(p<0.001)$, age (ANOVA, $p<0.05$, controls were older on average), but no differences in gender (Chi Squared, $p=0.70$ ), years of education ( $p=0.42, A N O V A$ ), or age at onset of symptoms for $\mathrm{MCl}$ and $\mathrm{AD}$ cohorts $(\mathrm{p}=0.79$, ttest).

PFwm was significantly associated with cohort (Figure $2 ; t=5.08, d f=424, p=5.6 e-7$ ), head motion RMS $(t=12.65, d f=424, p=2 e-16)$ and global $R M S(t=6.16, d f=424, p=1.68 e-9)$, whereas age $(p=0.075)$ and site $(p=0.062)$ were associated with non-significant trends. The effects of visit $(p=0.51)$ and WM volume $(p=0.31)$ were not significant.

Adjusted PFwm was associated with regional glucose metabolism (Figure 3A; $\mathrm{t}=-\mathrm{-2.93}$, $d f=96, p=0.0042)$ but not ventricular volume $(p=0.49)$ nor hippocampal volume $(p=0.44)$. Note these data were extracted from samples that had all of these neuroimaging measures available to permit a direct comparisons with PFwm. Adjusted PFwm was not associated with any of the CSF biomarkers ( $p>0.73$ with $d f=63$ ). For the cognitive composite scores, adjusted PFwm was correlated with composite memory (Figure 3B; $t=-2.69$, $d f=148, p=0.0079$ ) but not composite executive function $(t=-0.97, d f=148, p=0.34)$. In the final regression model that included cohort, glucose metabolism, composite memory and head and global RMS 
variables, cohort and glucose metabolism were significant $(t=2.71, p=0.0078$, and $t=-2.07$, $p=0.040$, respectively for $d f=117)$, as were head displacement $R M S$ and global $R M S(t=11.23$, $p=2 e-16$, and $t=6.97, p=2 e-10$, respectively for $d f=117$ ), while composite memory was not $(p=0.63)$.

\section{Discussion}

Resting state $\mathrm{fMRI}$ data from ADNI were used to investigate physiological fluctuations in white matter as a source of cerebrovascular contrast in three cognitive groups. We found support for our primary hypothesis: PFwm was significantly increased in AD compared to the $\mathrm{MCl}$ and control groups. Secondary regression analyses were conducted on subsets of ADNI participants to support the relevance of PFwm to established biomarkers. PFwm was inversely correlated with regional grey matter glucose metabolism from the neuroimaging data, and inversely correlated with the memory from the cognitive composite score data. Glucose metabolism and cohort remained as significantly associated with PFwm, whereas memory was not, when each of these variables were included in one omnibus model.

Multiple pathophysiological sources could contribute to our findings of increased PFwm in AD. Amyloid angiopathy and tortuous arterioles in white matter are reported in $A D$ and this could explain why PFwm was increased in the current sample of AD participants ${ }^{44}$. Mechanistically, altered cerebrovascular compliance ${ }^{45}$ and/or increased transmission of arterial pulsatility effects into white matter could explain elevated PFwm ${ }^{17,21}$, which is consistent with previous findings on intracranial pulsatility ${ }^{15,16}$. While these physiological phenomena may not be purely $A D$ etiology, mixed $A D$ and cerebrovascular disease is recognized as the most common clinical dementia and raises questions about the role of cerebrovascular dysfunction in cognitive decline. Indeed, Scuteri et al. reported that systemic arterial stiffness was a strong 
predictor of cognitive decline among older adults with memory problems ${ }^{11}$. Microvascular aberrations such as thickening of the basement membrane, luminal narrowing and endothelial compression decrease vascular compliance ${ }^{46}$, which would contribute to propagation of higher pulsatility down the vascular system and into tissue. A future direction for the current study is to consider PFwm in the context of diffusion tensor imaging (DTI), however, both DTI and rs-fMRI are unfortunately not available for the same participants in ADNI. Such an analysis would speak to white matter changes, such as demyelination, axonal and tissue volume changes that occur in $A D$ and could influence rs-fMRI white matter signals ${ }^{45}$.

Results of our secondary analyses suggest that PFwm may have merit as a new and complimentary imaging biomarker to study neurodegeneration. Although intracranial pulsatility is reported to be associated with increased ventricular volume ${ }^{15}$, we failed to detect a significant association of PFwm with ventricular or with hippocampal volume. Whereas, we did find that PFwm was negatively associated with glucose metabolism in the Landau $\mathrm{ROI}{ }^{26}$, which are regions that continue to be implicated in $A D{ }^{47}$. Both the correlation analysis of adjusted PFwm with the biomarkers and the full model stepwise backward regression demonstrate that adjusted PFwm provide unique information with respect to cohort. We believe this was the case because PFwm provides physiological information that is not captured by the four biomarkers that were tested in this study, hence the between cohort differences in PFwm may reflect underlying differences in cerebrovascular health. Furthermore, brain pulsatility can be characterized by various means and has demonstrated diagnostic merit in neurodegenerative research ${ }^{11,15,16}$.

It is important to discuss limitations of the current study. First, we have previously shown that it is ideal to isolate cardiac pulsatility effects on rs-fMRI, particularly among adults with diffuse white matter hyperintensities ${ }^{17}$, however this requires high temporal resolution rs-fMRI to 
sample the cardiac signals that was not part of the ADNI rs-fMRI. Second, despite our previous findings ${ }^{17}$, we did not include white matter hyperintensity $(\mathrm{WMH})$ lesion volume in the current study. Although WMH small vessel disease has been linked to vascular pulsatility measures ${ }^{10}$, at the time of this study the ADNI sample appears to exhibit low WMH burden levels ${ }^{48}$, thus future considerations are warranted. Third, cardiac monitoring by pulse oximetry was also not part of the ADNI rs-fMRI data collection. This limits our ability to characterise PFwm in relation to heart rate during rs-fMRI. Similarly, it remains to be seen whether the respiratory cycle influences PFwm. Fourth, while the sample size for our primary objective was large, our secondary analyses were performed on subsets of participants because of the need for multiple datasets (e.g. samples with rs-fMRI and FDG PET). This also speaks to the challenges of considering longitudinal analysis of the ADNI data since at the time of this study there were insufficient rs-fMRI scans beyond one year. It would be useful to replicate our findings from the secondary analysis as ADNI data accumulates. Furthermore, it would be useful to consider rs-fMRI data beyond one year in ADNI, which was an inclusion criteria by necessity because of the smaller number of samples and the need to address retention bias, specifically in the AD cohort.

In summary, this study demonstrates that physiological fluctuations can be extracted from white matter regions in rs-fMRI data and used to study neurodegeneration. PFwm was robustly different between the cognitive cohorts and was associated with established neuroimaging and cognitive biomarkers of AD. PFwm may therefore be useful in characterising the vascular complications associated with AD. 


\section{Acknowledgements}

This study was funded by the Canadian Institutes of Health Research (CIHR CSE133351).

Data collection and sharing for this project was funded by the Alzheimer's Disease

Neuroimaging Initiative (ADNI) (National Institutes of Health Grant U01 AG024904) and DOD ADNI (Department of Defense award number W81XWH-12-2-0012). ADNI is funded by the National Institute on Aging, the National Institute of Biomedical Imaging and Bioengineering, and through generous contributions from the following: Alzheimer's Association; Alzheimer's Drug Discovery Foundation; Araclon Biotech; BioClinica, Inc.; Biogen Idec Inc.; Bristol-Myers Squibb Company; Eisai Inc.; Elan Pharmaceuticals, Inc.; Eli Lilly and Company; Eurolmmun; F. Hoffmann-La Roche Ltd and its affiliated company Genentech, Inc.; Fujirebio; GE Healthcare; IXICO Ltd.; Janssen Alzheimer Immunotherapy Research \& Development, LLC.; Johnson \& Johnson Pharmaceutical Research \& Development LLC.; Medpace, Inc.; Merck \& Co., Inc.; Meso Scale Diagnostics, LLC.; NeuroRx Research; Neurotrack Technologies; Novartis Pharmaceuticals Corporation; Pfizer Inc; Piramal Imaging; Servier; Synarc Inc; and Takeda Pharmaceutical Company. The CIHR is providing funds to support ADNI clinical sites in Canada. Private sector contributions are facilitated by the Foundation for the National Institutes of Health. The grantee organization is the Northern California Institute for Research and Education, and the study is coordinated by the Alzheimer's Disease Cooperative Study at the University of California, San Diego. ADNI data were disseminated by the Laboratory for Neuro Imaging at the University of Southern California. We would also like to thank all volunteers and their families for their time and commitment. Without them, ADNI could not exist. 


\section{References}

1. Petersen RC, Doody R, Kurz A, et al. Current concepts in mild cognitive impairment. Archives of neurology. Dec 2001;58(12):1985-1992.

2. Sperling RA, Aisen PS, Beckett LA, et al. Toward defining the preclinical stages of Alzheimer's disease: recommendations from the National Institute on Aging-Alzheimer's Association workgroups on diagnostic guidelines for Alzheimer's disease. Alzheimer's \& dementia : the journal of the Alzheimer's Association. May 2011;7(3):280-292.

3. Ganguli M, Snitz BE, Saxton JA, et al. Outcomes of mild cognitive impairment by definition: a population study. Archives of neurology. Jun 2011;68(6):761-767.

4. Nettiksimmons J, DeCarli C, Landau S, Beckett L, Alzheimer's Disease Neuroimaging I. Biological heterogeneity in ADNI amnestic mild cognitive impairment. Alzheimer's \& dementia : the journal of the Alzheimer's Association. Sep 2014;10(5):511-521 e511.

5. Lee MJ, Seo SW, Na DL, et al. Synergistic effects of ischemia and beta-amyloid burden on cognitive decline in patients with subcortical vascular mild cognitive impairment. JAMA psychiatry. Apr 2014;71(4):412-422.

6. Thal DR, Rub $U$, Orantes $M$, Braak $H$. Phases of A beta-deposition in the human brain and its relevance for the development of AD. Neurology. Jun 25 2002;58(12):1791-1800.

7. Vinters HV, Secor DL, Pardridge WM, Gray F. Immunohistochemical study of cerebral amyloid angiopathy. III. Widespread Alzheimer A4 peptide in cerebral microvessel walls colocalizes with gamma trace in patients with leukoencephalopathy. Annals of neurology. Jul 1990;28(1):34-42.

8. Bell RD, Winkler EA, Singh I, et al. Apolipoprotein E controls cerebrovascular integrity via cyclophilin A. Nature. May 24 2012;485(7399):512-516.

9. Honjo K, Black SE, Verhoeff NP. Alzheimer's disease, cerebrovascular disease, and the beta-amyloid cascade. The Canadian journal of neurological sciences. Le journal canadien des sciences neurologiques. Nov 2012;39(6):712-728.

10. Mitchell GF, van Buchem MA, Sigurdsson S, et al. Arterial stiffness, pressure and flow pulsatility and brain structure and function: the Age, Gene/Environment Susceptibility--Reykjavik study. Brain : $a$ journal of neurology. Nov 2011;134(Pt 11):3398-3407.

11. Scuteri A, Tesauro M, Appolloni S, Preziosi F, Brancati AM, Volpe M. Arterial stiffness as an independent predictor of longitudinal changes in cognitive function in the older individual. Journal of hypertension. May 2007;25(5):1035-1040.

12. Johnson NA, Jahng GH, Weiner MW, et al. Pattern of cerebral hypoperfusion in Alzheimer disease and mild cognitive impairment measured with arterial spin-labeling MR imaging: initial experience. Radiology. Mar 2005;234(3):851-859.

13. Alsop DC, Casement M, de Bazelaire C, Fong T, Press DZ. Hippocampal hyperperfusion in Alzheimer's disease. Neurolmage. Oct 1 2008;42(4):1267-1274.

14. O'Rourke MF, Hashimoto J. Mechanical factors in arterial aging: a clinical perspective. Journal of the American College of Cardiology. Jul 3 2007;50(1):1-13.

15. Wahlin A, Ambarki K, Birgander R, Malm J, Eklund A. Intracranial pulsatility is associated with regional brain volume in elderly individuals. Neurobiology of aging. Feb 2014;35(2):365-372.

16. Bateman GA, Levi CR, Schofield P, Wang Y, Lovett EC. The venous manifestations of pulse wave encephalopathy: windkessel dysfunction in normal aging and senile dementia. Neuroradiology. Jun 2008;50(6):491-497.

17. Makedonov I, Black SE, Macintosh BJ. BOLD fMRI in the white matter as a marker of aging and small vessel disease. PloS one. 2013;8(7):e67652.

18. Zhou J, Greicius MD, Gennatas ED, et al. Divergent network connectivity changes in behavioural variant frontotemporal dementia and Alzheimer's disease. Brain : a journal of neurology. May 2010;133(Pt 5):1352-1367.

19. Fox MD, Zhang D, Snyder AZ, Raichle ME. The global signal and observed anticorrelated resting state brain networks. Journal of neurophysiology. Jun 2009;101(6):3270-3283. 
20. Chai XJ, Castanon AN, Ongur D, Whitfield-Gabrieli S. Anticorrelations in resting state networks without global signal regression. Neurolmage. Jan 16 2012;59(2):1420-1428.

21. Dagli MS, Ingeholm JE, Haxby JV. Localization of cardiac-induced signal change in fMRI. Neurolmage. Apr 1999;9(4):407-415.

22. Shmueli $K$, van Gelderen $P$, de Zwart JA, et al. Low-frequency fluctuations in the cardiac rate as a source of variance in the resting-state fMRI BOLD signal. Neurolmage. Nov 1 2007;38(2):306-320.

23. Wang $\mathrm{HH}$, Menezes NM, Zhu MW, et al. Physiological noise in MR images: an indicator of the tissue response to ischemia? Journal of magnetic resonance imaging : JMRI. Apr 2008;27(4):866-871.

24. Jahanian H, Ni WW, Christen T, Moseley ME, Kurella Tamura M, Zaharchuk G. Spontaneous BOLD signal fluctuations in young healthy subjects and elderly patients with chronic kidney disease. PloS one. 2014;9(3):e92539.

25. Ding Z, Newton AT, Xu R, Anderson AW, Morgan VL, Gore JC. Spatio-temporal correlation tensors reveal functional structure in human brain. PloS one. 2013;8(12):e82107.

26. Landau SM, Harvey D, Madison CM, et al. Associations between cognitive, functional, and FDG-PET measures of decline in AD and MCl. Neurobiology of aging. Jul 2011;32(7):1207-1218.

27. Nestor SM, Rupsingh R, Borrie M, et al. Ventricular enlargement as a possible measure of Alzheimer's disease progression validated using the Alzheimer's disease neuroimaging initiative database. Brain : $a$ journal of neurology. Sep 2008;131(Pt 9):2443-2454.

28. Jack CR, Jr., Petersen RC, O'Brien PC, Tangalos EG. MR-based hippocampal volumetry in the diagnosis of Alzheimer's disease. Neurology. Jan 1992;42(1):183-188.

29. Crane PK, Carle A, Gibbons LE, et al. Development and assessment of a composite score for memory in the Alzheimer's Disease Neuroimaging Initiative (ADNI). Brain imaging and behavior. Dec 2012;6(4):502-516.

30. Shaw LM, Vanderstichele H, Knapik-Czajka M, et al. Cerebrospinal fluid biomarker signature in Alzheimer's disease neuroimaging initiative subjects. Annals of neurology. Apr 2009;65(4):403-413.

31. McKhann G, Drachman D, Folstein M, Katzman R, Price D, Stadlan EM. Clinical diagnosis of Alzheimer's disease: report of the NINCDS-ADRDA Work Group under the auspices of Department of Health and Human Services Task Force on Alzheimer's Disease. Neurology. Jul 1984;34(7):939-944.

32. McKhann GM, Knopman DS, Chertkow H, et al. The diagnosis of dementia due to Alzheimer's disease: recommendations from the National Institute on Aging-Alzheimer's Association workgroups on diagnostic guidelines for Alzheimer's disease. Alzheimer's \& dementia : the journal of the Alzheimer's Association. May 2011;7(3):263-269.

33. Petersen RC, Smith GE, Waring SC, Ivnik RJ, Tangalos EG, Kokmen E. Mild cognitive impairment: clinical characterization and outcome. Archives of neurology. Mar 1999;56(3):303-308.

34. Folstein MF, Folstein SE, McHugh PR. "Mini-mental state". A practical method for grading the cognitive state of patients for the clinician. Journal of psychiatric research. Nov 1975;12(3):189-198.

35. D. W. Wechsler Memory Scale San Antonio, TX Psychol. Corp.; 1997.

36. Morris JC. The Clinical Dementia Rating (CDR): current version and scoring rules. Neurology. Nov 1993;43(11):2412-2414.

37. Jack CR, Jr., Bernstein MA, Fox NC, et al. The Alzheimer's Disease Neuroimaging Initiative (ADNI): MRI methods. Journal of magnetic resonance imaging : JMRI. Apr 2008;27(4):685-691.

38. Triantafyllou C, Hoge RD, Krueger G, et al. Comparison of physiological noise at $1.5 \mathrm{~T}, 3 \mathrm{~T}$ and $7 \mathrm{~T}$ and optimization of fMRI acquisition parameters. Neurolmage. May 15 2005;26(1):243-250.

39. Sled JG, Zijdenbos AP, Evans AC. A nonparametric method for automatic correction of intensity nonuniformity in MRI data. IEEE transactions on medical imaging. Feb 1998;17(1):87-97.

40. Jenkinson $M$, Bannister $P$, Brady $M$, Smith $S$. Improved optimization for the robust and accurate linear registration and motion correction of brain images. Neurolmage. Oct 2002;17(2):825-841.

41. Zuo XN, Kelly C, Adelstein JS, Klein DF, Castellanos FX, Milham MP. Reliable intrinsic connectivity networks: test-retest evaluation using ICA and dual regression approach. Neurolmage. Feb 1 2010;49(3):2163-2177. 
42. Satterthwaite TD, Elliott MA, Gerraty RT, et al. An improved framework for confound regression and filtering for control of motion artifact in the preprocessing of resting-state functional connectivity data. Neurolmage. Jan 1 2013;64:240-256.

43. Power JD, Barnes KA, Snyder AZ, Schlaggar BL, Petersen SE. Spurious but systematic correlations in functional connectivity MRI networks arise from subject motion. Neurolmage. Feb 1 2012;59(3):21422154.

44. Brown WR, Moody DM, Thore CR, Anstrom JA, Challa VR. Microvascular changes in the white mater in dementia. Journal of the neurological sciences. Aug 15 2009;283(1-2):28-31.

45. Thomas BP, Liu P, Park DC, van Osch MJ, Lu H. Cerebrovascular reactivity in the brain white matter: magnitude, temporal characteristics, and age effects. Journal of cerebral blood flow and metabolism : official journal of the International Society of Cerebral Blood Flow and Metabolism. Feb 2014;34(2):242247.

46. de la Torre JC. Alzheimer disease as a vascular disorder: nosological evidence. Stroke; a journal of cerebral circulation. Apr 2002;33(4):1152-1162.

47. Langbaum JB, Chen K, Lee W, et al. Categorical and correlational analyses of baseline fluorodeoxyglucose positron emission tomography images from the Alzheimer's Disease Neuroimaging Initiative (ADNI). Neurolmage. May 1 2009;45(4):1107-1116.

48. Ramirez J, McNeely AA, Scott CJ, Masellis M, Black SE, Alzheimer's Disease Neuroimaging I. White matter hyperintensity burden in elderly cohort studies. The Sunnybrook Dementia Study, Alzheimer Disease Neuroimaging Initiative, and Three-City Study. Alzheimer's \& dementia : the journal of the Alzheimer's Association. Jul 212015. 


\section{Table 1}

\begin{tabular}{|c|c|c|c|c|}
\hline & Controls & $\mathrm{MCl}$ & AD & $\mathrm{P}$ value \\
\hline Participants & 53 & 102 & 34 & \\
\hline Total rs-fMRI datasets & 117 & 246 & 74 & \\
\hline Average visits per participant & 2.2 & 2.1 & 2.2 & \\
\hline Age at baseline (years, mean $\pm s d$ ) & $75.5 \pm 6.7$ & $71.9 \pm 7.7$ & $72.5 \pm 7.1$ & $<0.05$ \\
\hline Gender (M/F) & $32 / 21$ & $49 / 53$ & $16 / 18$ & 0.70 \\
\hline Education (years \pm sd) & $16.5 \pm 2.2$ & $16.1 \pm 2.6$ & $15.8 \pm 2.9$ & 0.42 \\
\hline Onset of symptoms (years \pm sd) & - & $67.5 \pm 8.1$ & $67.1 \pm 8.0$ & 0.79 \\
\hline MMSE (mean of all time points \pm sd) & $28.9 \pm 1.5$ & $27.4 \pm 2.2$ & $21.5 \pm 3.5$ & $<0.001$ \\
\hline Clinical Dementia Rating (median) & 0 & 0.5 & 1 & $<0.001$ \\
\hline $\begin{array}{l}\text { Functional Assessment Questionnaire } \\
(\text { FAQ) (mean } \pm \mathrm{sd})\end{array}$ & $0.5 \pm 2.4$ & $3.6 \pm 5.0$ & $16.0 \pm 7.1$ & $<0.001$ \\
\hline Cerebrospinal Fluid (sub-sample size) & 16 & 47 & 5 & \\
\hline Amyloid-beta level (mean \pm sd) & $174.8 \pm 45.0$ & $173.8 \pm 58.2$ & $126.9 \pm 13.9$ & 0.23 \\
\hline Tau level (mean \pm sd) & $71.2 \pm 37.4$ & $94.0 \pm 60.1$ & $130.4 \pm 70.2$ & 0.04 \\
\hline Phosphorylated tau level (mean $\pm \mathrm{sd}$ ) & $33.4 \pm 11.9$ & $45.0 \pm 25.2$ & $62.0 \pm 22.5$ & 0.01 \\
\hline
\end{tabular}

Table 1. Sample characteristics. 


\section{Figure Captions}

Figure 1. PFwm colour maps in axial view for three representative participants in each cohort and overlaid on a group template T1-weighted image. Top row: controls, middle row: $\mathrm{MCl}$, bottom row: AD. The right column shows the average physiological fluctuation maps by cohort.

Figure 2. PFwm boxplots are plotted by cohort and visit (within Year 1). Controls (blue), $\mathrm{MCl}$ (green) and AD (red) are shown by different colour bars. PFwm values are in units of \% BOLD signal change and are adjusted for head displacement and global signal root-mean square BOLD signal (global RMS). Visits were centered at baseline, 3 and 6 months using kmeans clustering. Cohort was a significant predictor of adjusted PFwm $(\mathrm{t}=5.08, \mathrm{df}=424$, $\left.\mathrm{p}=6 \times 10^{-7}\right)$.

Figure 3. Scatterplots were used to visualise regression results from single visit data. PFwm (adjusted for head displacement and global RMS) is plotted against A) FDG glucose metabolism ( $\mathrm{t}=-2.93, \mathrm{df}=96, \mathrm{p}=0.004$, intensity normalization $\mathrm{FDG}$ ) and $\mathrm{B}$ ) the composite memory score $(\mathrm{t}=-2.69, \mathrm{df}=148, \mathrm{p}=0.008)$. 


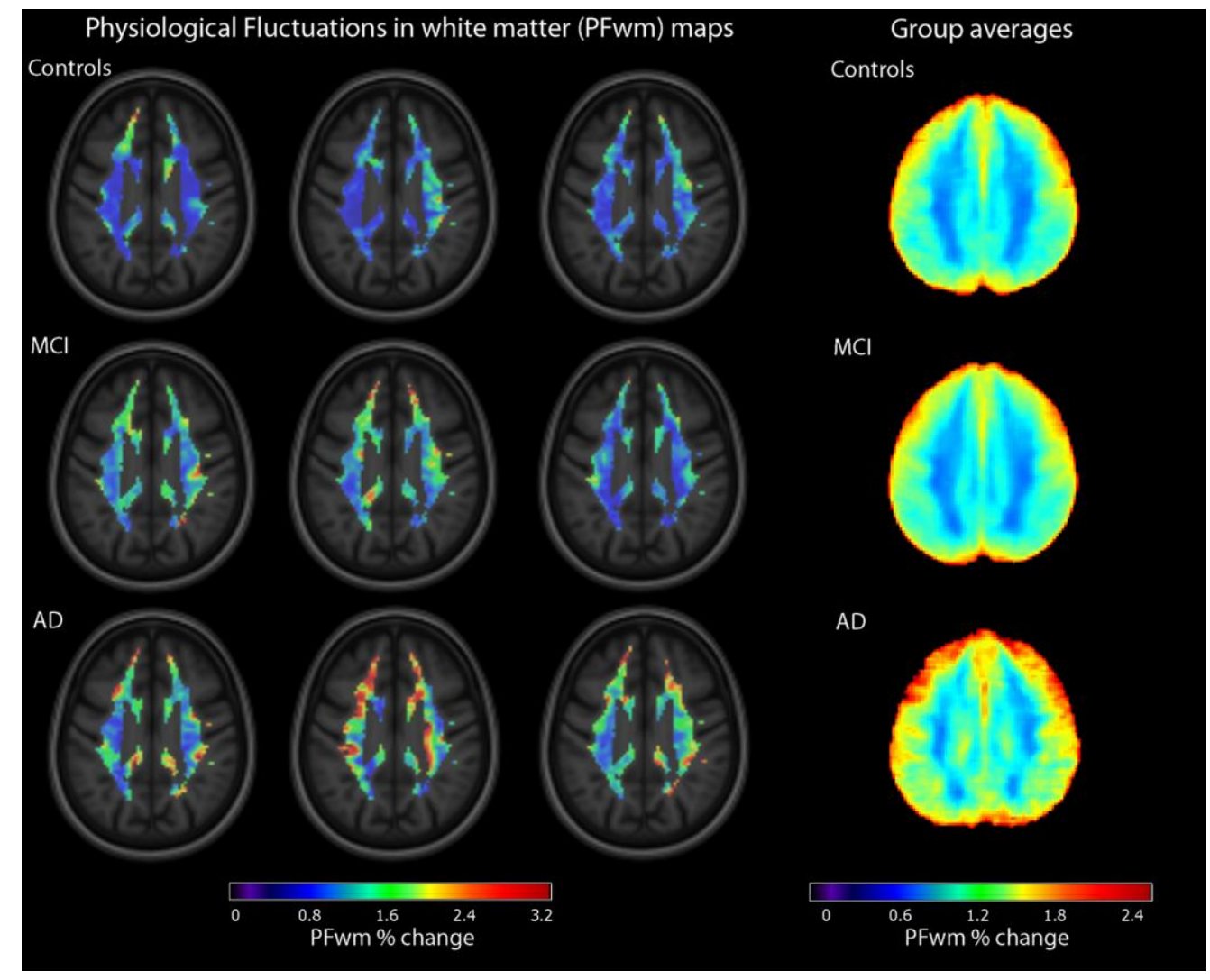




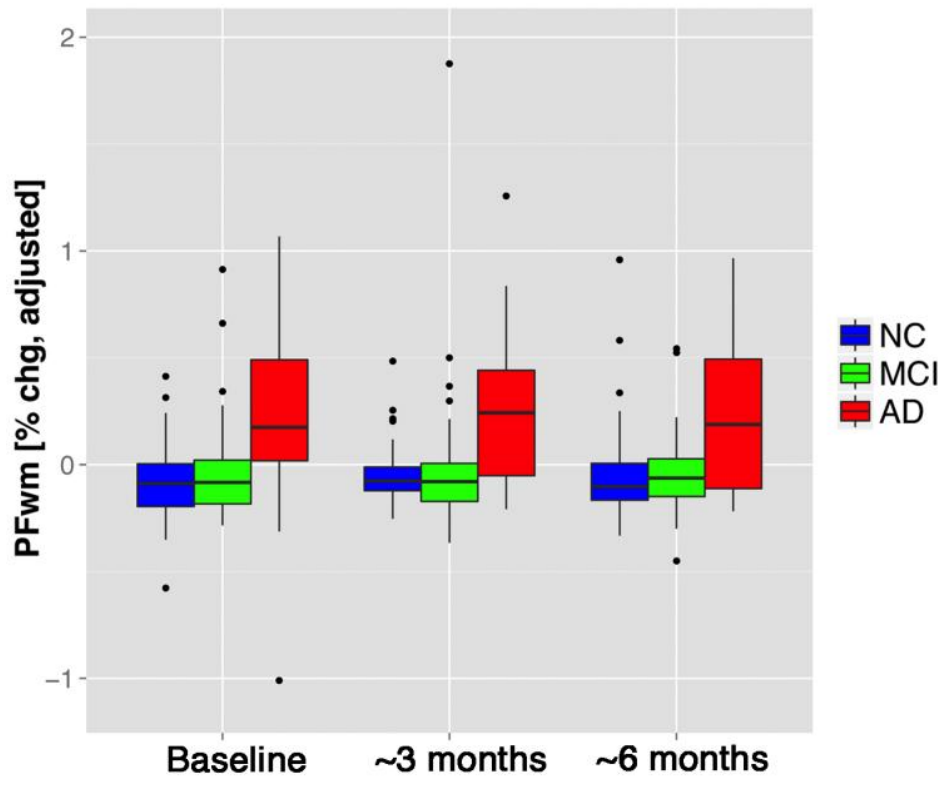



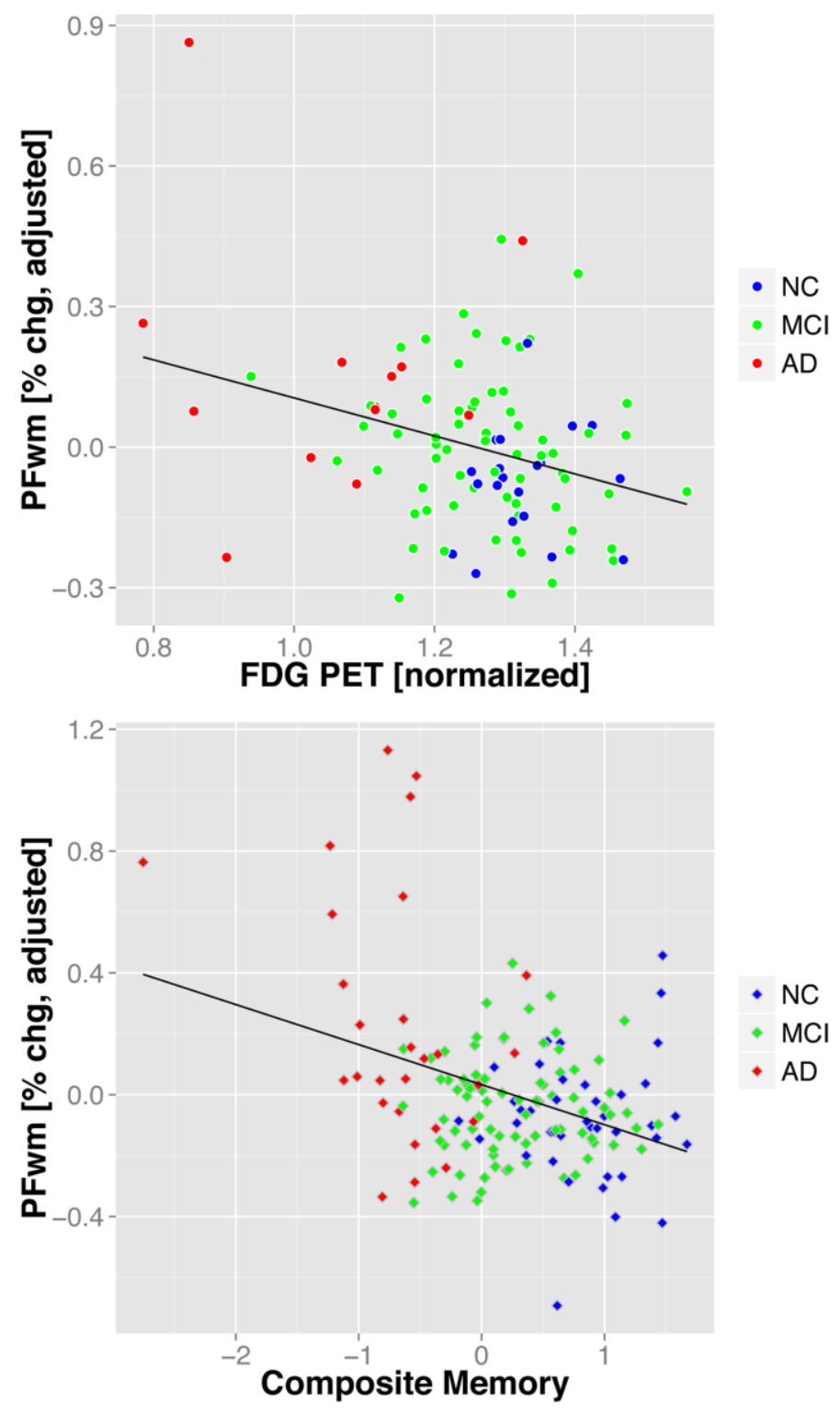